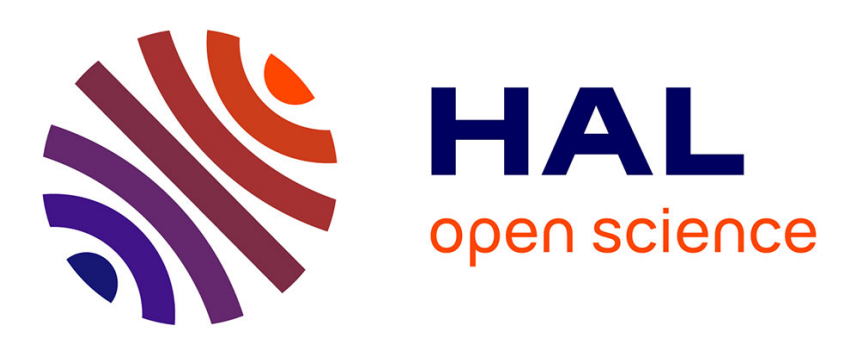

\title{
Detecting Alzheimer's disease using machine learning methods
}

Kia Dashtipour, William Taylor, Shuja Ansari, Adnan Zahid, Mandar Gogate, Jawad Ahmad, Khaled Assaleh, Kamran Arshad, Muhammad Ali Imran, Qammer Abbai

\section{To cite this version:}

Kia Dashtipour, William Taylor, Shuja Ansari, Adnan Zahid, Mandar Gogate, et al.. Detecting Alzheimer's disease using machine learning methods. EAI, Oct 2021, Glasgow, United Kingdom. hal-03381752

\section{HAL Id: hal-03381752 \\ https://hal.science/hal-03381752}

Submitted on 17 Oct 2021

HAL is a multi-disciplinary open access archive for the deposit and dissemination of scientific research documents, whether they are published or not. The documents may come from teaching and research institutions in France or abroad, or from public or private research centers.
L'archive ouverte pluridisciplinaire HAL, est destinée au dépôt et à la diffusion de documents scientifiques de niveau recherche, publiés ou non, émanant des établissements d'enseignement et de recherche français ou étrangers, des laboratoires publics ou privés. 


\title{
Detecting Alzheimer's disease using machine learning methods
}

\author{
Kia Dashtipour ${ }^{1,3}$, William Taylor ${ }^{1}$, Shuja Ansari ${ }^{1}$, Adnan Zahid ${ }^{2}$, Mandar \\ Gogate $^{3}$, Jawad Ahmad ${ }^{3}$, Khaled Assaleh ${ }^{4}$, Kamran Arshad ${ }^{4}$, Muhammad Ali \\ $\operatorname{Imran}^{1,5}$, and Qammer Abbai ${ }^{1}$ \\ 1.James Watt School of Engineering, University of Glasgow,Glasgow, United \\ Kingdom kia.dashtipour@glasgow.ac.uk \\ 2. School of Engineering and Physical Science, Heriot-Watt University; Edinburgh, \\ EH144AS, UK \\ 3. School of Computing, Edinburgh Napier University, Edinburgh, U.K. \\ 4. Faculty of Engineering and IT, Ajman University, Ajman 346, UAE 5. Artificial \\ Intelligence Research Center (AIRC), Ajman University, Ajman, UAE
}

\begin{abstract}
As the world is experiencing population growth, the portion of the older people, aged 65 and above, is also growing at a faster rate. As a result, the dementia with Alzheimer's disease is expected to increase rapidly in the next few years. Currently, healthcare systems require an accurate detection of the disease for its treatment and prevention. Therefore, it has become essential to develop a framework for early detection of Alzheimer's disease to avoid complications. To this end, a novel framework, based on machine-learning (ML) and deep-learning (DL) methods, is proposed to detect Alzheimer's disease. In particular, the performance of different ML and DL algorithms has been evaluated against their detection accuracy. The experimental results state that bidirectional long short-term memory (BiLSTM) outperforms the ML methods with a detection accuracy of $91.28 \%$. Furthermore, the comparison with the stateof-the-art indicates the superiority of the our framework over the other proposed approaches in the literature.
\end{abstract}

Keywords: Machine learning · Deep learning · Detecting Alzheimer.

\section{Introduction}

Alzheimer is from a family of diseases that can develop dementia, specially in elderly people. Dementia is a loss of memory and/or other mental disability that can cause physical damaged to the brain. Although Alzheimer is the most common type of dementia but there are different types of dementia [35, 49, such as vascular dementia, Lewy Body disease, frontotemporal dementia, alcohol related dementia and HIV associated dementia, etc. The most common type of dementia after Alzheimer's disease is vascular dementia which can happens after stroke. In addition, some of the causes of dementia are reversible such as thyroid problem and vitamin deficiencies. The dementia is not just a disease but its 
associated risks such as decline in the memory significantly reduces a person's ability to perform daily tasks. It is expected that the number of people affected from dementia will increase over the time. The early detection can not only help doctors to precisely make decision on the treatment but also help preventing the complications 21]. It is important to develop a system that can help in early detection of dementia.

The Alzheimer's disease has number of symptoms, especially in the elderly people that can cause problems to perform daily tasks due to memory loss. Although the Alzheimer is not normal due to aging, its risk factor increases with the aging. Most of the people who suffer from Alzheimer are aged 65 or above. However, it not uncommon to have this disease in the people younger than 65. For instance, more than two hundred thousand American aged less than 65 suffers from Alzheimer disease. Fig 1 shows the difference between the normal brain and Alzheimer's brain [47.

It can be noticed that the brain of the Alzheimer's disease in not only significantly smaller than the normal brain but is affected severely from neurological disorder and dysfunction. Additionally, Fig 2 presents some of the common symptoms of the Alzheimer's disease. The most common types of symptoms are loss of memory, changes in the behaviour, difficulty with everyday task and confusion in familiar environments.

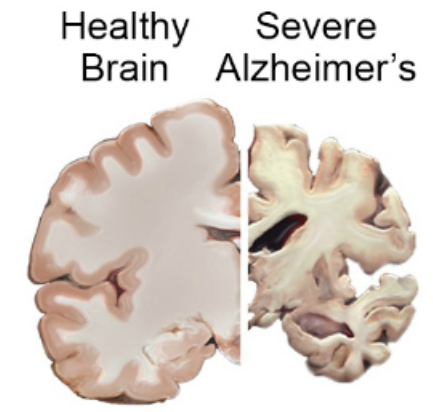

Fig. 1. Difference between a normal brain and a severe Alzheimer's Brain 47].

Practically, no effective cure to treat Alzheimer's disease exist to date. However, there exist ways that can temporarily slow down the process of Alzheimer's symptoms and improve the quality of the life of the patient. To this end, significant research efforts are dedicated to find the effective ways of treating the Alzheimer's disease with a focus on preventing the disease from progressing over the time 39 .

It is suggested that ML and DL algorithms, which have proven their significance in various fields, can help solve the problem of early detection of Alzheimer's disease clearly, ML and DL methods have their applications in var- 


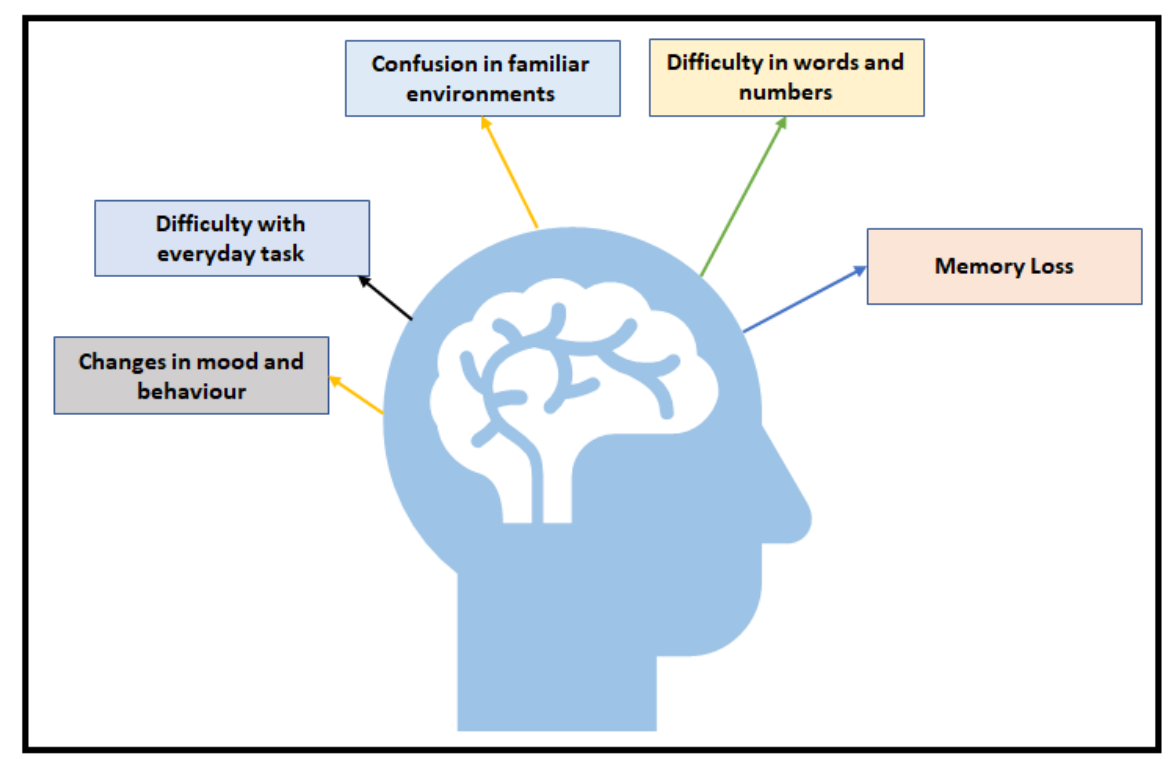

Fig. 2. Alzheimer symptoms

ious domains, including but not limited to sentiment analysis $2,911,13,19$, 30, 32, 33], speech enhancement 27, 28], cyber-security 31, 34, image classification [36], energy efficiency [51, travel detection [5, 6], posture detection [48, and atrial fibrillation [37, 38], etc. Therefore, the ML techniques including support vector machine (SVM), logistic regression, multi-layered perceptron and deep learning classifiers. In particular, feature selection is an important element of traditional ML classifiers which is inherently incorporated in DL classifiers. Generally, DL classifiers achieve better results on large datasets.

In this paper, we proposed a novel approach, based on ML and DL methods, to detect Alzheimer's disease. The obtained results from DL algorithm are compared against traditional ML algorithms $1,24,26$. In particular, bidirectional long short-term memory (BiLSTM) outperforms all the considered ML and DL methods, with a detection accuracy of $91.28 \%$.

The rest of the paper is structured as follows. Section 2 provides the stateof-the-art in Alzheimer detection. Section 3 presents the proposed methodology. Section 4 provides the experimental results of the proposed Alzheimer detection and discussion, and finally Section 5 concludes the paper.

\section{Related Work}

In this section, we discuss the current state-of-the-art to detect dementia and Alzheimer's disease using DL and ML algorithms.

The work in 7 propose novel metrics to identify the Alzheimer's disease using pattern similarity score. The authors characterize the metrics in terms 
of conditional probabilities modeled by logistic regression. In addition, they explore the performance of anatomical and cognitive impairment which is used to generate the output of the classifiers using different types of data.

The authors in 41] use the online available datasets of MRI scan images and other cognitive features, such as RAVLT tests, MOCA and FDG score etc to identify the Alzheimer's disease. In particular, clustering algorithms are developed based on logistic regression and SVM to detect the patient having Alzheimer's disease. Ammar et al. 4] presented a framework based on the speech processing to detect dementia. The framework was used to extract features from patients with dementia and without dementia wherein the speech data used was having verbal description and manual transcription. Therefore, the speech and textual features were used to train ML classifiers. The authors achieved an overall accuracy of $79 \%$ only.

Another interesting work in presented in [52] where authors introduced a detection method based on the MRI images of brain based on the Eigenbrain. Their approach used SVM and particle swarm optimization to train the model. Their proposal achieved satisfactory results in detecting the parts of the brain affected from Alzheimer's disease. Working on the similar lines of MRI scans, authors in [44] detected dementia and other different features using gradient boost and Artificial Neural Network (ANN) models. The authors achieved comparable results with the ones presented in [52]. The authors in [45] proposed a hybrid multimodal method based on the cognitive and linguistic features. The authors used ANN to train the model detect Alzheimer's disease and its severity. Their scheme achieved good results as compared to the state-of-the-art.

\section{$3 \quad$ Methodology}

This work proposes a novel Alzheimer's detection system using different ML and DL algorithms. In particular, the raw data data coming from MRI scans is pre-processed before applying various ML and DL methods. Fig 3 presents an overall picture of the proposed Alzheimer detection system.

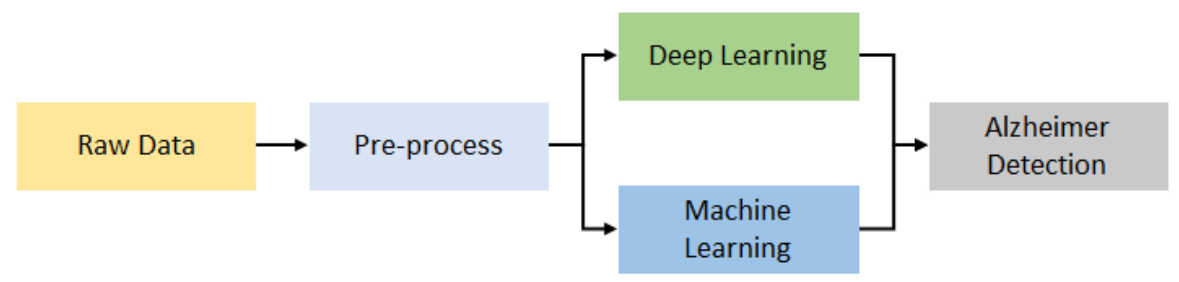

Fig. 3. Overview of Alzheimer Detection Framework 


\subsection{Machine Learning Methods}

This subsection highlights our simulation settings to train different ML models. Scikit-learn is used to train ML classifiers. More specifically, radial basis function (RBF) kernel is used to train support vector machine (SVM). Elasticnet is used as a penalty for logistic regression, two hidden layers are used for multilayer perceptron (MLP), number of neighbors are set to 5 for k-nearest neighbors (KNN), epsilon is set to float for Naïve Bayes, max features are set to int for decision tree and finally number of estimate is set to 100 for random forest. It is worth mentioning that the ML classifiers are trained based on standard deviation, average, square root, skew, maximum and minimum value.

\subsection{Deep Learning Methods}

This subsection highlights our simulation settings to train different DL models. Mainly, two different DL models are used in this work, namely convolutional neural network (CNN) and LSTM The developed CNN architecture, inspired from $12,29,42$, contains input, hidden and output layers where hidden layers are made up of convolutional, max pooling and fully connected layers. In particular, 10-layered CNN architecture is employed. On the other hand, LSTM architecture, inspired from [3, 8, 23, 43, contains two bidirectional LSMT along with 128 and 64 cells with dropout of 0.2 . In addition, a dense layer with two neurons and softmax activation is used.

\section{Experimental results and discussions}

The dataset consists of 373 images from 150 subjects aged between 60 and 96 . The MRI scan of each subject was taken for his one or two visits with a separation of at least one year between visits. All the subjects were right-handed with a mixture of men and women. Out of 150,72 subjects were non-demented, with no mental disorder or dysfunction. On the other hand, 64 subjects were categorized as demented during their initial visit, including 51 with mild to moderate Alzheimer's disease. Importantly, the dataset is marked with five labels as normal, very mild dementia, mild dementia, moderate dementia, severe dementia.

In order to detect Alzheimer's disease, we compare the results of different ML classifiers including logistic regression, SVM, random forest, MLP, KNN, naïve bayes, decision tree and DL classifiers (1D-CNN, 2D-CNN, LSTM and BiLSTM). For ML classifiers, the features, such as skew, percentile, standard deviation, mean and square root are used to train the classifier. However, for DL classifiers raw data is used to train the models. It is important to that we used 5 -fold and 10-fold cross-validation to perform the experiments. The considered evaluation parameters are precision, recall, f-measure and detection accuracy. It is evident from Tables 1 highlights are results of different ML and DL methods for a 5-fold cross validation settings. It can be noted that SVM provides the most promising results as compared to other ML methods such as logistic regression, 


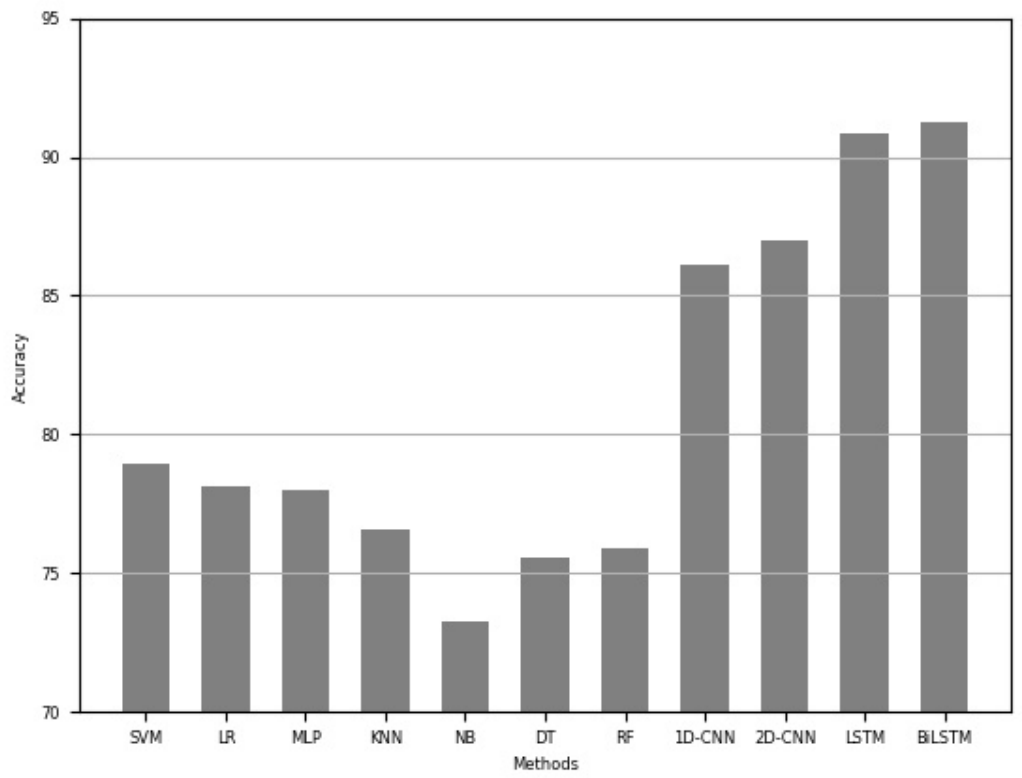

Fig. 4. 5-Fold Summary of Machine learning and deep learning Results

MLP and etc. Overall, the experimental results show that the DL classifiers outperforms ML methods. However, the DL classifiers are expensive both in terms of computational resources and time.

Table 2 shows the summary of considered ML and DL results to the detect Alzheimer's disease for a 10-fold cross validation settings. Here again, SVM classifiers outperforms to other ML approaches such as logistic regression, MLP, KNN, Naive Bayes, decision tree and random forest. On the the other hand, naive bayes gives the worst performance as compared to all ML and DL methods and also it took longer to train the model. Overall, DL methods perform better. In particular, BiLSTM achieved the best performance. However, BiLSTM took longer to train the model.

\subsection{Discussion}

It is important to note that the detection of Alzheimer's disease using ML methods is cost-effective (Computation and time) than DL algorithms. On the other hand, training deep learning classifiers is time and computationally expensive. Clearly, as shown in Figs 4 and 5 the BiLSTM achieved better performance as compared to other methods in both 5 -fold and 10 -fold cross validation strategies. In addition to having such a promising results, our work has certain limitations 


\begin{tabular}{|l|l|l|l|l|l|l|}
\hline Methods & $\begin{array}{l}\text { Training } \\
\text { Accuracy }\end{array}$ & $\begin{array}{l}\text { Testing } \\
\text { Accuracy }\end{array}$ & Precision & Recall & F-Score & Time \\
\hline SVM & 80.78 & 78.56 & 0.78 & 0.76 & 0.76 & $2 \mathrm{~m} \mathrm{21} \mathrm{s}$ \\
\hline $\begin{array}{l}\text { Logistic } \\
\text { Regression }\end{array}$ & 80.23 & 78.12 & 0.78 & 0.77 & 0.78 & $2 \mathrm{~m} 8 \mathrm{~s}$ \\
\hline MLP & 79.81 & 78 & 0.78 & 0.77 & 0.78 & $2 \mathrm{~m} 6 \mathrm{~s}$ \\
\hline KNN & 77.25 & 76.58 & 0.76 & 0.75 & 0.76 & $2 \mathrm{~m} \mathrm{~s}$ \\
\hline Naive Bayes & 75.89 & 73.28 & 0.73 & 0.72 & 0.73 & $2 \mathrm{~m} 19 \mathrm{~s}$ \\
\hline Decision Tree & 76.98 & 75.59 & 0.75 & 0.74 & 0.75 & $2 \mathrm{~m} 11 \mathrm{~s}$ \\
\hline Random Forest & 76.85 & 75.89 & 0.75 & 0.75 & 0.75 & $2 \mathrm{~m} 31 \mathrm{~s}$ \\
\hline 1D-CNN & 88.59 & 86.14 & 0.86 & 0.85 & 0.86 & $8 \mathrm{~m} 28 \mathrm{~s}$ \\
\hline 2D-CNN & 89.45 & 87 & 0.87 & 0.86 & 0.87 & $9 \mathrm{~m} 1 \mathrm{~s}$ \\
\hline LSTM & 91.26 & 90.85 & 0.90 & 0.90 & 0.90 & $10 \mathrm{~m} 17 \mathrm{~s}$ \\
\hline BiLSTM & 93.21 & 91.28 & 0.91 & 0.91 & 0.91 & $10 \mathrm{~m} 12 \mathrm{~s}$ \\
\hline
\end{tabular}

Table 1. Summary of Machine learning and deep learning methods to detect Alzheimer using 5 -fold cross-validation

\begin{tabular}{|c|c|c|c|c|c|c|}
\hline Methods & $\begin{array}{l}\text { Training } \\
\text { Accuracy }\end{array}$ & $\begin{array}{l}\text { Testing } \\
\text { Accuracy }\end{array}$ & Precision & Recall & F-Score & Time \\
\hline SVM & 82.24 & 80.75 & 0.80 & 0.79 & 0.80 & $2 \mathrm{~m} 33 \mathrm{~s}$ \\
\hline $\begin{array}{l}\text { Logisitc } \\
\text { Regression }\end{array}$ & 81.86 & 79.86 & 0.79 & 0.78 & 0.79 & $2 \mathrm{~m} 12 \mathrm{~s}$ \\
\hline MLP & 80.36 & 79.56 & 0.79 & 0.79 & 0.79 & $2 \mathrm{~m} 31 \mathrm{~s}$ \\
\hline KNN & 78.91 & 76.12 & 0.76 & 0.75 & 0.76 & $2 \mathrm{~m} 4 \mathrm{~s}$ \\
\hline Naive Bayes & 75.2 & 71.64 & 0.71 & 0.70 & 0.71 & $3 \mathrm{~m}$ \\
\hline Decision Tree & 78.69 & 75.9 & 0.75 & 0.74 & 0.75 & $2 \mathrm{~m} 19 \mathrm{~s}$ \\
\hline Random Forest & 75.97 & 73.29 & 0.73 & 0.72 & 0.73 & $2 \mathrm{~m} 8 \mathrm{~s}$ \\
\hline 1D-CNN & 88.91 & 86.54 & 0.86 & 0.85 & 0.86 & $8 \mathrm{~m} 5 \mathrm{~s}$ \\
\hline 2D-CNN & 89.43 & 87.01 & 0.87 & 0.86 & 0.87 & $8 \mathrm{~m} 29 \mathrm{~s}$ \\
\hline LSTM & 93.19 & 91.19 & 0.91 & 0.91 & 0.91 & $8 \mathrm{~m} 45 \mathrm{~s}$ \\
\hline BiLSTM & 95.59 & 93.19 & 0.93 & 0.93 & 0.93 & $9 \mathrm{~m} 16 \mathrm{~s}$ \\
\hline
\end{tabular}

Table 2. Summary of Machine learning and deep learning methods to detect Alzheimer using 10-fold cross-validation

\begin{tabular}{|l|l|l|l|l|l|}
\hline Ref & Accuracy & Precision & Recall & F-Score \\
\hline Zhang et al. & & 86.24 & 0.85 & 0.83 & 0.84 \\
\hline Dyrba et al. & & 70.4 & 0.70 & 0.70 & 0.70 \\
\hline Escudero et al. & & 79.1 & 0.78 & 0.76 & 0.75 \\
\hline Trambaiolli et al. & $\mathbf{5 0}$ & 75.56 & 0.75 & 0.73 & 0.71 \\
\hline Liu et al. 40. & $84 . .40$ & 0.84 & 0.82 & 0.82 \\
\hline Shankar et al. & 46 & 76.23 & 0.75 & 0.74 & 0.75 \\
\hline Our Approach & 93.19 & 0.93 & 0.93 & 0.93 \\
\hline
\end{tabular}

Table 3. Comparison with State-of-the-art approach 


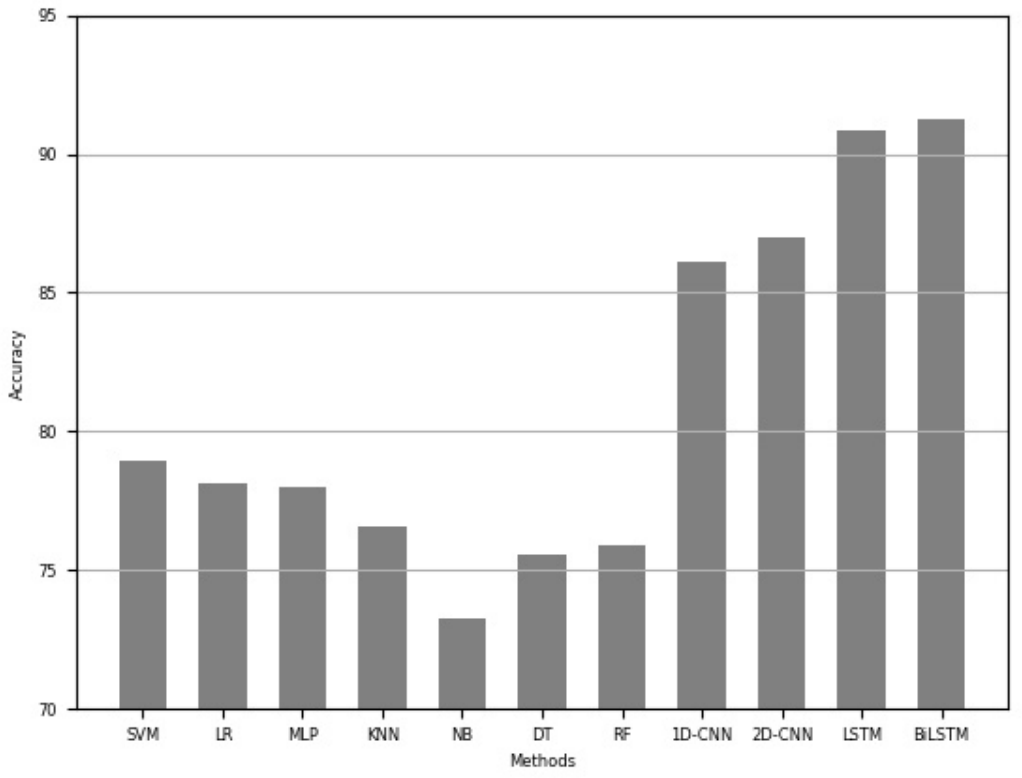

Fig. 5. 5-Fold Summary of Machine learning and deep learning Results

as well (1) The dataset is very small with only 373 images in total. (2) The dataset considers people of aged 65 and above only.

\section{Conclusion}

The alzheimer's disease is the most challenging health problems scientists are facing since decades. In this paper, we present a novel framework based on the ML and DL algorithms including SVM, logistic regression, MLP, KNN, Naive Bayes, decision tree and random forest, 1D-CNN, 2D,CNN, LSTM and BiLSTM to automatically detect Alzheimer's disease. The extensive experimental results show that BiLSTM achieved better performance as compared to other ML and DL algorithms. As a future work, we intend to use transformers to detect Alzheimer's disease using images, visual and acoustic features.

\section{Acknowledgement}

This work is supported in part by the Ajman University Internal Research Grant. 


\section{References}

1. Ahsan Adeel, Mandar Gogate, and Amir Hussain. Contextual deep learning-based audio-visual switching for speech enhancement in real-world environments. Information Fusion, 59:163-170, 2020.

2. Rami Ahmed, Mandar Gogate, Ahsen Tahir, Kia Dashtipour, Bassam Al-Tamimi, Ahmad Hawalah, Mohammed A El-Affendi, and Amir Hussain. Deep neural network-based contextual recognition of arabic handwritten scripts. Entropy, 23(3):340, 2021.

3. Abdulrahman S Alqarafi, Ahsan Adeel, Mandar Gogate, Kia Dashitpour, Amir Hussain, and Tariq Durrani. Toward's arabic multi-modal sentiment analysis. In International Conference in Communications, Signal Processing, and Systems, pages 2378-2386. Springer, 2017.

4. Randa Ben Ammar and Yassine Ben Ayed. Speech processing for early alzheimer disease diagnosis: machine learning based approach. In 2018 IEEE/ACS 15th International Conference on Computer Systems and Applications (AICCSA), pages 1-8. IEEE, 2018.

5. Syed Muhammad Asad, Shuja Ansari, Metin Ozturk, Rao Naveed Bin Rais, Kia Dashtipour, Sajjad Hussain, Qammer H Abbasi, and Muhammad Ali Imran. Mobility management-based autonomous energy-aware framework using machine learning approach in dense mobile networks. Signals, 1(2):170-187, 2020.

6. Syed Muhammad Asad, Kia Dashtipour, Sajjad Hussain, Qammer Hussain Abbasi, and Muhammad Ali Imran. Travelers-tracing and mobility profiling using machine learning in railway systems. In 2020 International Conference on UKChina Emerging Technologies (UCET), pages 1-4. IEEE, 2020.

7. Ramon Casanova, Fang-Chi Hsu, Kaycee M Sink, Stephen R Rapp, Jeff D Williamson, Susan M Resnick, Mark A Espeland, Alzheimer's Disease Neuroimaging Initiative, et al. Alzheimer's disease risk assessment using large-scale machine learning methods. PloS one, 8(11):e77949, 2013.

8. Andrew Churcher, Rehmat Ullah, Jawad Ahmad, Fawad Masood, Mandar Gogate, Fehaid Alqahtani, Boubakr Nour, William J Buchanan, et al. An experimental analysis of attack classification using machine learning in iot networks. Sensors, 21(2):446, 2021.

9. Kia Dashtipour, Mandar Gogate, Ahsan Adeel, Abdulrahman Algarafi, Newton Howard, and Amir Hussain. Persian named entity recognition. In 2017 IEEE 16th International Conference on Cognitive Informatics 83 Cognitive Computing (ICCI* $C C)$, pages 79-83. IEEE, 2017.

10. Kia Dashtipour, Mandar Gogate, Ahsan Adeel, Amir Hussain, Abdulrahman Alqarafi, and Tariq Durrani. A comparative study of persian sentiment analysis based on different feature combinations. In International Conference in Communications, Signal Processing, and Systems, pages 2288-2294. Springer, 2017.

11. Kia Dashtipour, Mandar Gogate, Ahsan Adeel, Cosimo Ieracitano, Hadi Larijani, and Amir Hussain. Exploiting deep learning for persian sentiment analysis. In International conference on brain inspired cognitive systems, pages 597-604. Springer, 2018.

12. Kia Dashtipour, Mandar Gogate, Ahsan Adeel, Hadi Larijani, and Amir Hussain. Sentiment analysis of persian movie reviews using deep learning. Entropy, 23(5):596, 2021.

13. Kia Dashtipour, Mandar Gogate, Erik Cambria, and Amir Hussain. A novel context-aware multimodal framework for persian sentiment analysis. arXiv preprint arXiv:2103.02636, 2021. 
14. Kia Dashtipour, Mandar Gogate, Jingpeng Li, Fengling Jiang, Bin Kong, and Amir Hussain. A hybrid persian sentiment analysis framework: Integrating dependency grammar based rules and deep neural networks. Neurocomputing, 380:1-10, 2020.

15. Kia Dashtipour, Amir Hussain, and Alexander Gelbukh. Adaptation of sentiment analysis techniques to persian language. In International Conference on Computational Linguistics and Intelligent Text Processing, pages 129-140. Springer, 2017.

16. Kia Dashtipour, Amir Hussain, Qiang Zhou, Alexander Gelbukh, Ahmad YA Hawalah, and Erik Cambria. Persent: a freely available persian sentiment lexicon. In International Conference on Brain Inspired Cognitive Systems, pages 310-320. Springer, 2016.

17. Kia Dashtipour, Soujanya Poria, Amir Hussain, Erik Cambria, Ahmad YA Hawalah, Alexander Gelbukh, and Qiang Zhou. Multilingual sentiment analysis: state of the art and independent comparison of techniques. Cognitive computation, 8(4):757-771, 2016.

18. Kia Dashtipour, Ali Raza, Alexander Gelbukh, Rui Zhang, Erik Cambria, and Amir Hussain. Persent 2.0: Persian sentiment lexicon enriched with domain-specific words. In International Conference on Brain Inspired Cognitive Systems, pages 497-509. Springer, 2019.

19. Kia Dashtipour, William Taylor, Shuja Ansari, Mandar Gogate, Adnan Zahid, Yusuf Sambo, Amir Hussain, Qammer Abbasi, and Muhammad Imran. Public perception towards fifth generation of cellular networks $(5 \mathrm{~g})$ on social media. Frontiers in Big Data, 2021.

20. Martin Dyrba, Michael Ewers, Martin Wegrzyn, Ingo Kilimann, Claudia Plant, Annahita Oswald, Thomas Meindl, Michela Pievani, Arun LW Bokde, Andreas Fellgiebel, et al. Robust automated detection of microstructural white matter degeneration in alzheimer's disease using machine learning classification of multicenter dti data. PloS one, 8(5):e64925, 2013.

21. Michael R D'Andrea, Gregory M Cole, and March D Ard. The microglial phagocytic role with specific plaque types in the alzheimer disease brain. Neurobiology of aging, 25(5):675-683, 2004.

22. Javier Escudero, Emmanuel Ifeachor, John P Zajicek, Colin Green, James Shearer, Stephen Pearson, Alzheimer's Disease Neuroimaging Initiative, et al. Machine learning-based method for personalized and cost-effective detection of alzheimer's disease. IEEE transactions on biomedical engineering, 60(1):164-168, 2012.

23. Alexander RT Gepperth, Thomas Hecht, and Mandar Gogate. A generative learning approach to sensor fusion and change detection. Cognitive Computation, 8(5):806-817, 2016.

24. Mandar Gogate, Ahsan Adeel, and Amir Hussain. Deep learning driven multimodal fusion for automated deception detection. In 2017 IEEE Symposium Series on Computational Intelligence (SSCI), pages 1-6. IEEE, 2017.

25. Mandar Gogate, Ahsan Adeel, and Amir Hussain. A novel brain-inspired compression-based optimised multimodal fusion for emotion recognition. In 2017 IEEE Symposium Series on Computational Intelligence (SSCI), pages 1-7. IEEE, 2017.

26. Mandar Gogate, Ahsan Adeel, Ricard Marxer, Jon Barker, and Amir Hussain. Dnn driven speaker independent audio-visual mask estimation for speech separation. arXiv preprint arXiv:1808.00060, 2018.

27. Mandar Gogate, Kia Dashtipour, Ahsan Adeel, and Amir Hussain. Cochleanet: A robust language-independent audio-visual model for real-time speech enhancement. Information Fusion, 63:273-285, 2020. 
28. Mandar Gogate, Kia Dashtipour, and Amir Hussain. Visual speech in real noisy environments (vision): A novel benchmark dataset and deep learning-based baseline system. Proc. Interspeech 2020, pages 4521-4525, 2020.

29. Mandar Gogate, Amir Hussain, and Kaizhu Huang. Random features and random neurons for brain-inspired big data analytics. In 2019 International Conference on Data Mining Workshops (ICDMW), pages 522-529. IEEE, 2019.

30. Imane Guellil, Ahsan Adeel, Faical Azouaou, Fodil Benali, Ala-Eddine Hachani, Kia Dashtipour, Mandar Gogate, Cosimo Ieracitano, Reza Kashani, and Amir Hussain. A semi-supervised approach for sentiment analysis of arab (ic+ izi) messages: Application to the algerian dialect. SN Computer Science, 2(2):1-18, 2021.

31. Zil E Huma, Shahid Latif, Jawad Ahmad, Zeba Idrees, Anas Ibrar, Zhuo Zou, Fehaid Alqahtani, and Fatmah Baothman. A hybrid deep random neural network for cyberattack detection in the industrial internet of things. IEEE Access, 9:5559555605, 2021.

32. Amir Hussain, Ahsen Tahir, Zain Hussain, Zakariya Sheikh, Mandar Gogate, Kia Dashtipour, Azhar Ali, and Aziz Sheikh. Artificial intelligence-enabled analysis of uk and us public attitudes on facebook and twitter towards covid-19 vaccinations. medRxiv, 2020.

33. Intisar O Hussien, Kia Dashtipour, and Amir Hussain. Comparison of sentiment analysis approaches using modern arabic and sudanese dialect. In International Conference on Brain Inspired Cognitive Systems, pages 615-624. Springer, 2018.

34. Cosimo Ieracitano, Ahsan Adeel, Mandar Gogate, Kia Dashtipour, Francesco Carlo Morabito, Hadi Larijani, Ali Raza, and Amir Hussain. Statistical analysis driven optimized deep learning system for intrusion detection. In International Conference on Brain Inspired Cognitive Systems, pages 759-769. Springer, 2018.

35. Cosimo Ieracitano, Annunziata Paviglianiti, Nadia Mammone, Mario Versaci, Eros Pasero, and Francesco Carlo Morabito. Socnnet: An optimized sobel filter based convolutional neural network for sem images classification of nanomaterials. In Progresses in Artificial Intelligence and Neural Systems, pages 103-113. Springer, 2021.

36. Fengling Jiang, Bin Kong, Jingpeng Li, Kia Dashtipour, and Mandar Gogate. Robust visual saliency optimization based on bidirectional markov chains. Cognitive Computation, pages 1-12, 2020.

37. Sidrah Liaqat, Kia Dashtipour, Kamran Arshad, and Naeem Ramzan. Non invasive skin hydration level detection using machine learning. Electronics, 9(7):1086, 2020.

38. Sidrah Liaqat, Kia Dashtipour, Adnan Zahid, Khaled Assaleh, Kamran Arshad, and Naeem Ramzan. Detection of atrial fibrillation using a machine learning approach. Information, 11(12):549, 2020.

39. J Lindeboom, B Schmand, L Tulner, G Walstra, and C Jonker. Visual association test to detect early dementia of the alzheimer type. Journal of Neurology, Neurosurgery \& Psychiatry, 73(2):126-133, 2002.

40. Siqi Liu, Sidong Liu, Weidong Cai, Sonia Pujol, Ron Kikinis, and Dagan Feng. Early diagnosis of alzheimer's disease with deep learning. In 2014 IEEE 11th international symposium on biomedical imaging (ISBI), pages 1015-1018. IEEE, 2014.

41. Priyanka Lodha, Ajay Talele, and Kishori Degaonkar. Diagnosis of alzheimer's disease using machine learning. In 2018 Fourth International Conference on Computing Communication Control and Automation (ICCUBEA), pages 1-4. IEEE, 2018. 
42. Shibli Nisar, Muhammad Tariq, Ahsan Adeel, Mandar Gogate, and Amir Hussain. Cognitively inspired feature extraction and speech recognition for automated hearing loss testing. Cognitive Computation, 11(4):489-502, 2019.

43. Metin Ozturk, Mandar Gogate, Oluwakayode Onireti, Ahsan Adeel, Amir Hussain, and Muhammad A Imran. A novel deep learning driven, low-cost mobility prediction approach for $5 \mathrm{~g}$ cellular networks: The case of the control/data separation architecture (cdsa). Neurocomputing, 358:479-489, 2019.

44. Ram Murti Rawat, Mohammad Akram, Setti Suneel Pradeep, et al. Dementia detection using machine learning by stacking models. In 2020 th International Conference on Communication and Electronics Systems (ICCES), pages 849-854. IEEE, 2020.

45. Utkarsh Sarawgi, Wazeer Zulfikar, Nouran Soliman, and Pattie Maes. Multimodal inductive transfer learning for detection of alzheimer's dementia and its severity. arXiv preprint arXiv:2009.00700, 2020.

46. K Shankar, SK Lakshmanaprabu, Ashish Khanna, Sudeep Tanwar, Joel JPC Rodrigues, and Nihar Ranjan Roy. Alzheimer detection using group grey wolf optimization based features with convolutional classifier. Computers \& Electrical Engineering, 77:230-243, 2019.

47. Kathleen Taylor. Dementia: A Very Short Introduction. Oxford University Press, 2020.

48. William Taylor, Syed Aziz Shah, Kia Dashtipour, Adnan Zahid, Qammer H Abbasi, and Muhammad Ali Imran. An intelligent non-invasive real-time human activity recognition system for next-generation healthcare. Sensors, 20(9):2653, 2020.

49. H Tohgi, T Abe, M Kimura, M Saheki, and S Takahashi. Cerebrospinal fluid acetylcholine and choline in vascular dementia of binswanger and multiple small infarct types as compared with alzheimer-type dementia. Journal of neural transmission, 103(10):1211-1220, 1996.

50. Lucas R Trambaiolli, Ana C Lorena, Francisco J Fraga, Paulo AM Kanda, Renato Anghinah, and Ricardo Nitrini. Improving alzheimer's disease diagnosis with machine learning techniques. Clinical EEG and neuroscience, 42(3):160-165, 2011.

51. Zheqi Yu, Pedro Machado, Adnan Zahid, Amir M Abdulghani, Kia Dashtipour, Hadi Heidari, Muhammad A Imran, and Qammer H Abbasi. Energy and performance trade-off optimization in heterogeneous computing via reinforcement learning. Electronics, 9(11):1812, 2020.

52. Yudong Zhang, Zhengchao Dong, Preetha Phillips, Shuihua Wang, Genlin Ji, Jiquan Yang, and Ti-Fei Yuan. Detection of subjects and brain regions related to alzheimer's disease using $3 \mathrm{~d}$ mri scans based on eigenbrain and machine learning. Frontiers in computational neuroscience, 9:66, 2015. 